\title{
An Instance of Somatoform Disorder
}

\author{
Armando Simon \\ Flying Phoenix, Live Oak, TX, USA \\ Email: CountNomis@aol.com
}

Received November 4, 2012; revised December 20, 2012; accepted December 29, 2012

\begin{abstract}
A case study is presented of a prison inmate with Somatoform Disorder, a relatively rare type of mental illness. In this particular case symptomatology revolved around his claim that some of his teeth were rotten, resulting in great pain, in spite of several diagnoses by dentists. When said teeth were extracted, inmate would express satisfaction, whereupon several weeks later the same complaint would resurface and the cycle began anew. Of the various types of mental illnesses listed in the DSM, there are some that are infrequently seen [1,2]. One of these is Somatoform Disorder, a category of mental illness for which there is a paucity of research, partly due to diagnostic difficulties and controversies $[3,4]$ and we would like to present a case study of just such an instance.
\end{abstract}

Keywords: Therapy; Somatoform Disorder; Prison

\section{Background Information}

The patient, in question was a $33 \mathrm{y} / \mathrm{o}$ black male recidivist, incarcerated in a multiunit state prison for burglary of a habitation. Patient $\mathrm{O}$, although usually talkative, was uncharacteristically evasive as to his personal background. Some of the information was obtained from sessions and other from previous documentation.

The patient finished his 7th grade education and obtained a GED while in a Missouri prison. He was the second of five siblings and his parents divorced when he was $5 \mathrm{y} / \mathrm{o}$. He stated that his father had had all of his teeth pulled out while one of his sister's had had several extracted. Although he denied any previous drug abuse history or any previous suicide attempt, records showed that he was hospitalized at the state hospitals in Arizona and Texas for alcoholism, the latter instance where he overdosed himself. He had nocturnal enuresis until the age of 11 and had his first trouble with the law at age 18 for stealing a car. He reported stuttering throughout his life, said stuttering being hardly detectable during interviews. Upon being asked if there had been any head trauma, patient $\mathrm{O}$ responded that he did not want to talk about it.

During his stay at the county jail and then later at the state prison, he received several disciplinary charges for minor, nonviolent infractions (e.g., insolence, disobeying a direct order, creating a disturbance).

\section{Mental Status}

There was no evidence of hallucination in patient $\mathrm{O}$. He was oriented as to time, place and person. He was well groomed, talkative and friendly. Long and short term memory was normal. His vocabulary and general knowledge were above average compared to the present population. Affect was normal and there did not appear to be any obvious damage to the central nervous system.

\section{Psychological Testing}

The 16 PF (Form C) was administered to the patient. The Motivational Distortion Scale $(\mathrm{MD}=8)$ shows that he attempted to put himself in a good light. A depressed C Scale 3 denoted an emotionally unstable individual with low tolerance for frustration, typical of prison inmates and many forms of neurotic disturbances. An elevated Q2 (8) denoted a temperamentally independent person uninterested in public opinion. And lastly, a depressed Q1 factor (2) denoted an attitude of tradition and conservatism.

The MMPI was also administered. The LFK validity scales were within the valid range, although the F-K result indicated a malingering attitude towards the test. Depressed scales were 5, 6 and 0 . The latter scale $(T=45)$ indicated an extroverted, though not necessarily gregarious, nature. Scale $6(T=38)$ is the low point of the profile indicating a highly suspicious, overly sensitive person with denial and projection for defense mechanisms. This was followed in elevation by Scale $5(T=46)$, while scales 3 and 4 were equivalent $(T=77)$ with a significant elevation for the neurotic triad (Scale $1(T=70)$; Scale 2 $(T=72)$; Scale $3(T=77))$.

\section{Presenting Problem}

The patient was first seen by the jail psychologist at the 
county level, the patient requesting that he be put in isolation in as much as he disliked other inmates. He complained that his teeth were hurting and was given a disciplinary report for accosting the jail captain demanding that a tooth be pulled. He did not request any other type of special treatment, such as being excused from work.

Upon arriving at the state prison, Patient $\mathrm{O}$ began to demand from prison officials that two or three of his teeth be pulled out, claiming that they were rotten, in so far as he was experiencing pain. The prison dentist, however, proclaimed them to be healthy and was, therefore, reluctant to extract them. Nevertheless, the patient was very insistent, claiming that he was the one experiencing the pain, so he knew what he was talking about. In various interviews, it came to light that before he had been incarcerated, he had gone to several dentists with the same request and complaint, moving to the next one if the teeth were not extracted.

Cognitive therapy was begun by the psychologist, but it rapidly became futile, as the patient was intractable in his demand.

At this point, it is necessary to insert the fact that the state prison system, at that time, was in hiatus. This stemmed from the federal government's insistence to reform the penal system. The state penal culture was experienceing an upheaval. Among the reforms instituted was the insistence that inmates be provided with adequate psychological and medical care, and that prisoners could file grievances against the staff if proper care was not carried out. Hitherto, both psychological and medical services had been subordinate to security concerns, even though at times there were no security issues involved and the inmates had been denied legitimate treatment. Several staff members had been terminated because they stubbornly refused to adjust to the new reality.

The prison dentist finally acquiesced, extracting two teeth, whereupon patient $\mathrm{O}$ proclaimed satisfaction, and being pain free. However, several weeks later, he would resume his complaints of pain in some of his teeth and demanded that they, too, be removed. After much hesitation and argumentation with the patient over the course of months, the very same pattern was repeated again and again until, finally, there were no more teeth to extract. At this point, he began to complain of pain in his jaw. He was transferred to a hospital unit for a neurological work- up and no physiological evidence was found for his complaint.

The unit psychiatrist began a psychotropic regimen, viz., prolixin. This resulted in the onset of tardive dyskenisia. The patient began to be less well groomed, some trembling becoming evident and his stuttering getting worse. At this point, the inmate was abruptly transferred to another unit for unrelated reasons and was not heard from again by the treatment team.

Although, ideally, one would like to present a case study with a beginning, middle and ending, particularly with a "happy ending," (especially for the beginning student) the truth of the matter is that oftentimes, for any number of reasons and because of circumstances beyond the clinician's power, there is no closure [5-7] and there is no "happy ending”. And that is something that a clinical student should also learn.

\section{REFERENCES}

[1] American Psychiatric Association, "Diagnostic and Statistical Manual of Mental Disorders,” American Psychiatric Association, Washington DC, 2005.

[2] S. Walsh and W. Denton, "Clinical Issues in Treating Somatoform Disorder for Couple Therapists," American Journal of Family Therapy, Vol. 33, No. 3, 2005, pp. 225-236. doi:10.1080/01926180590932979

[3] J. Dimsdale and F. Creed, "The Proposed Diagnosis of Somatic Symptom Disorders in DSM-V to Replace Somatoform Disorders in DSM-IV-A Preliminary Report," Journal of Psychosomatic Research, Vol. 66, No. 6, 2009, pp. 473-476. doi:10.1016/j.jpsychores.2009.03.005

[4] D. Lynch, A. McGrady, R. Nagel and C. Zsembik, "Somatization in Family Practice: Comparing 5 Methods of Classification,” Primary Care Companion Journal of Clinical Psychiatry, Vol. 1, No. 3, 1999, pp. 85-89.

[5] D. Frayn, "Assessment Factors Associated with Premature Termination,” American Journal of Psychotherapy, Vol. 46, No. 2, 1992, pp. 250-262.

[6] J. Ogrodniczuk, "Strategies for Reducing Patient-Initiated Premature Termination in Psychotherapy,” Harvard Review of Psychiatry, Vol. 13, No. 2, 2005, pp. 54-70. doi:10.1080/10673220590956429

[7] R. Westmacott and J. Hunsley, "Reasons for Terminating Psychotherapy,” Journal of Clinical Psychology, Vol. 66, No. 9, 2010, pp. 965-977. 